\title{
Volker Schütte Parteienfinanzierung: Die doppelte Wende des Bundesverfassungsgerichts und die Folgen
}

»Ein neues Grundgesetz der Parteienfinanzierung « hat das Bundesverfassungsgericht nach den Worten des GRÜNEN-Anwalts Uwe Günther der Bundesrepublik beschert ${ }^{1}$. In der Tat bedeutet dieses nunmehr sechste einschlägige Urteil vom 9.4. $1992^{2}$ einen unerwartet tiefen Einschnitt ins Parteien- wie vor allem auch ins Steuerrecht. Übereinstimmend bewerteten die Kommentatoren die Entscheidung als »Schuß vor den Bug« der Parteien, denen in Karlsruhe unmißverständlich ihre Grenzen aufgezeigt worden seien (»bis hierhin und nicht weiter «). Neue Konzepte seien bei den jetzt anstehenden Novellierungen mehr denn je gefragt. Für das Bundesverfassungsgericht selbst stellt das Urteil eine Korrektur seiner bisherigen Rechtsprechung zur Parteienfinanzierung dar. Der Zweite Senat hat dabei in doppelter Hinsicht eine Wende vollzogen: bei der Subventionierung der Parteien durch Spenden und bei der direkten Alimentierung der Parteien durch den Staat.

In dem von der Partei DIE GRÜNEN angestrengten Organstreitverfahren wurden im Ergebnis sowohl die vormals sanktionierte steuerliche Absetzbarkeit von Spenden juristischer Personen als auch die von Großspenden natürlicher Personen für verfassungswidrig erklärt. Außerdem setzte das Gericht die Publizitätsschwelle, ab der die Parteien Spenden in ihren Rechenschaftsberichten auszuweisen haben, von $40000 \mathrm{DM}$ auf $20000 \mathrm{DM}$ herab. Andererseits ist es dem Staat im Gegensatz zur bisherigen Rechtsprechung nicht länger verwehrt, die Parteien für ihre sich aus dem Grundgesetz ergebende Tätigkeit - unter Beachtung bestimmter Schranken - allgemein zu finanzieren. Die gleichfalls auf dem Prüfstand stehenden »Sockelbetrag « und »Chancenausgleich « wurden hingegen als mit dem Grundgesetz nicht vereinbar deklariert.

\section{Die Finanzierung der Parteien durch Spenden}

Im Jahr I 986 hatte das Bundesverfassungsgericht die steuerliche Begünstigung von Parteispenden bis zu einer Höhe von 100000 DM je Bürger und Jahr als verfassungsgemäß angesehen ${ }^{3}$, wobei Körperschaften das gleiche Recht konzediert bekamen. Diesen Spielraum nutzte der Gesetzgeber daraufhin - angesichts der vorgegebenen Geldsumme in fast bescheidener Zurückhaltung-, indem er zum I. I. I989 in $\$ 10 \mathrm{~b}$ Einkommensteuergesetz und $₫ 9 \mathrm{Nr} .3$ b) Körperschaftsteuergesetz die Obergrenzen für die steuerliche Absetzbarkeit von Parteispenden auf 60000 DM je Spender und Jahr festlegte. In der jüngsten Entscheidung vollzog das Bundesverfassungsgericht nun sechs Jahre später eine Kehrtwende ${ }^{4}$ und verwarf die steuerliche Begünstigung von Parteispenden juristischer Personen vollständig, diejenige natürlicher Personen mit Ausnahme des $\$ 34 \mathrm{~g}$ Einkommensteuergesetz (der sog. Kleinspendenregelung). Der jüngste Urteilsspruch weicht aber in den genannten Punkten nicht nur von der früheren Rechtsprechung ab, sondern knüpft daneben bewußt an die I 986 zu Protokoll gegebene abweichende Meinung der Richter Böckenförde und Mahrenholz

\footnotetext{
I Zit. n. Suddeutsche Zeitung vom ro. 4. 1992.

2 Zuerst abgedruckt in Europassche Grundrechte Zeitschrift (EuGRZ) 1992, 153 ff.

3 BVerfGE $73,40$.

4 EuGRZ $1992,153,167$ f.
} 
an', deren Minderheitsvotum der Zweite Senat sich insofern jetzt einstimmig anschloß. Im übrigen bedeutet die Begründung des Gerichts die Rückkehr zum Tenor des Parteienfinanzierungsurteils des Jahres $1958^{6}$.

Die angegriffenen Regelungen verletzten die Chancengleichheit der Parteien', weil sie "Parteien, die mit ihrem Programm einkommensstarke und insbesondere unternehmerisch tätige Bevölkerungskreise in besonderer Weise ansprechen «, bevorzugten, ohne daß der vom Gesetzgeber zur Kompensation geschaffene sog. Chancenausgleich ${ }^{8}$ diese Ungleichbehandlung der Parteien zu heilen imstande gewesen wäre9. Auf der anderen Seite verstießen die angesprochenen Steuervergünstigungen gegen den Grundsatz der gleichen Teilhabe der Bürger an der politischen Willensbildung ${ }^{10}$. Das Bundesverfassungsgericht geht dabei offenkundig vom Leitbild des Durchschnittsverdieners aus. Dieser könne aber den bisherigen Spendenrahmen nicht entfernt ausschöpfen, weil der sogar höher liege als das zur Verfügung stehende Einkommen der Bezugsperson ". Außerdem vertrage sich der bei der Ausübung politischer Rechte anzuwendende formale Gleichheitssatz ebenso nicht mit der Steuerbegünstigung für Parteispenden juristischer Personen, da hierdurch »denjenigen natürlichen Personen, die hinter ihnen stehen, eine zusätzliche Möglichkeit vom Staat und damit zu Lasten der übrigen Steuerzahler - geförderter Einflußnahme auf die politische Willensbildung"verschafft werde ${ }^{12}$.

Die Verfassungsrichter erkannten zudem in der Anhebung der Publizitätsgrenze von 20000 auf 40000 DM einen Verstoß gegen Artikel 2I I 4 GG ${ }^{13}$, da bei der einfachgesetzlichen Ausgestaltung der grundgesetzlichen Rechenschaftslegungspflicht auch die Auswirkungen von Parteispenden auf kommunaler Ebene und bei kleineren Parteien nicht aus dem Blick geraten dürften ${ }^{14}$. Überlegungen des Verfassungsgerichts, wie Umgehungen der Vorschrift (z. B. durch Zwischenschaltung von »Strohmännern * oder Mandatsträgern) zu verhindern wären, schließen ungeachtet der in $\$ 23 a$ und $\$ 2$ 25 Parteiengesetz bereits bestehenden Regelungen daran an ${ }^{15}$. Sollte denn die Vermutung begründet sein, daß nach nun erfolgter "Ächtung « der Unternehmerspenden durch das Bundesverfassungsgericht diese Zuwendungen (wie schon bis 1983) künftig wieder auf Umwegen ihre Empfänger in den Parteien erreichen werden? Letztlich ließ das in Rede stehende Urteil im Bereich des "Spendenwesens" der Parteien - abgesehen einmal von dessen prinzipieller Zulässigkeit - nämlich nur die Möglichkeit unangetastet, eine Parteispende (bzw. einen Mitgliedsbeitrag) von bis zu I 200 DM jährlich zur Hälfte direkt von der Steuerschuld abziehen zu können. Hier erklärte das Gericht eine Anpassung der Beträge an die Einkommensentwicklung für denkbar ${ }^{16}$, da bei dieser Zuwendungshöhe eine politische Einflußnahme nicht zu befürchten sei.

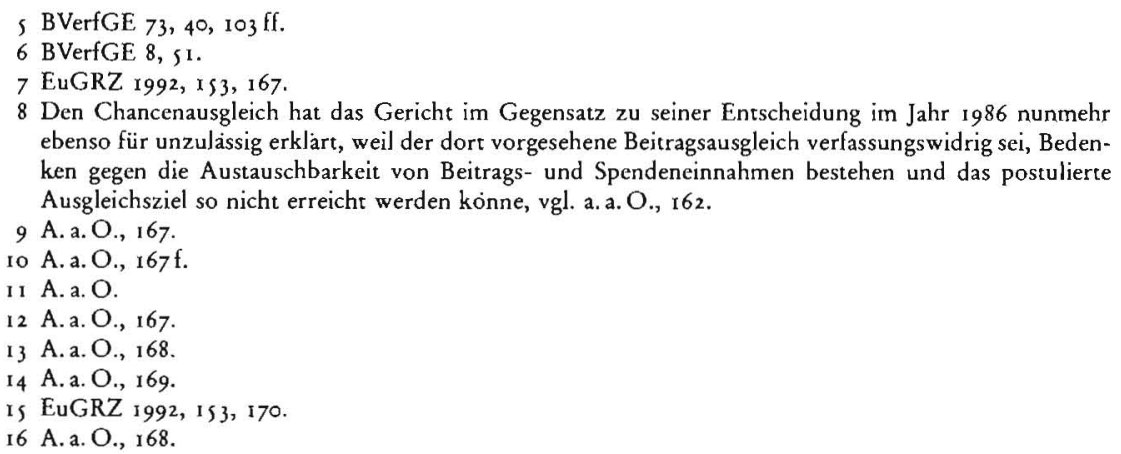


Seit 1968 war es Auffassung des Bundesverfassungsgerichts, daß der Staat den Parteien lediglich die »notwendigen Kosten eines angemessenen Wahlkampfes « erstatten dürfe ${ }^{17}$. Eine darüber hinausgehende öffentliche Alimentation gefährdete die vom Grundgesetz gebotene Freiheit der Parteien vom Staat, hätte diese frei gebildeten, in der Gesellschaft wurzelnden Organisationen in die Abhängigkeit staatlicher Vorsorge führen können ${ }^{18}$. Mit dieser Ansicht hat das Gericht jetzt gebrochen; im Rahmen noch näher zu skizzierender Grenzen ist in Zukunft eine generelle Staatsfinanzierung zugelassen ${ }^{19}$. Die Richter haben somit eine Fiktion, eine »mühsam aufrechterhaltene Theorie aufgegeben ${ }^{20}$, um nicht nur hehren Grundsätzen, sondern pragmatisch auch der Realität Rechnung zu tragen ${ }^{21}$. Außerdem hatte das Gericht ja schon seit langem die Pauschalierung der Wahlkampfkosten sowie im Rahmen der Erstattung Abschlagszahlungen bereits in den drei Jahren vor den Wahlen akzeptiert $^{22}$. Die Tätigkeit der Parteien lasse sich aus heutiger Sicht eben doch nicht auf die bloße Wahlvorbereitung reduzieren, und eine Abgrenzung dieses A ufgabenbereiches von den übrigen auf Artikel 2 I I I GG beruhenden Betätigungsfeldern könnte nur rein äußerlich vorgenommen werden ${ }^{23}$.

Auf den ersten Blick mag es da überraschen, daß das Gericht dennoch die sog. Sockelbetragsregelung mit sofortiger Wirkung suspendierte ${ }^{24}$. Die Richter lehnten diese "erfolgsunabhängige Basisfinanzierung " der Parteien ab, da der "Sockel «, dieses Ewigkeitspodest (wie Antje Vollmer ihn nannte), der Verankerung der Parteien in der Gesellschaft und der damit verbundenen Staatsferne zuwiderliefe ${ }^{25}$. Weil die politischen Parteien auch ökonomisch von der Zustimmung der Bevölkerung abhängig bleiben müßten, komme staatlicherseits ebenfalls nur eine partielle Finanzierung ihrer allgemeinen Tätigkeit in Betracht ${ }^{26}$. Der Wegfall der enggefaßten Zweckbindung für staatliche Mittel wird durch die Festschreibung von Obergrenzen relativiert $^{27}$. Das Bundesverfassungsgericht bestätigte $\$$ i 8 VII Parteiengesetz, wonach die staatlichen Zuwendungen an die Parteien in der Gesamtsumme nicht die der selbst erwirtschafteten Einnahmen übersteigen dürfen ("relative Obergrenze«). Außerdem bestimmte es den Jahresmittelwert der öffentlichen Subventionierung der Parteien in den Jahren 1989 bis 1992 als »absolute Obergrenze« der staatlichen Zuwendungen, wobei lediglich eine Anpassung an die Preisentwicklung gestattet sei. Unter dem Strich sei der Staat nach den Worten eines Kritikers trotz aller Einschränkungen "nunmehr endgültig als Futterkrippe für seine Träger etabliert « worden ${ }^{28}$. Diesem Verdikt kann jedoch nur eingeschränkt gefolgt werden, da der bereits früher vorgeschriebene fünfzigprozentige Eigenanteil der Parteien an ihrer Finanzierung endgültig nicht mehr die zur Manipulation der Bilanzen geeigneten »Einnahmen aus Krediten « umfassen darf ${ }^{29}$ und die Einkünfte aus dem Chancenausgleich aufgrund ihres

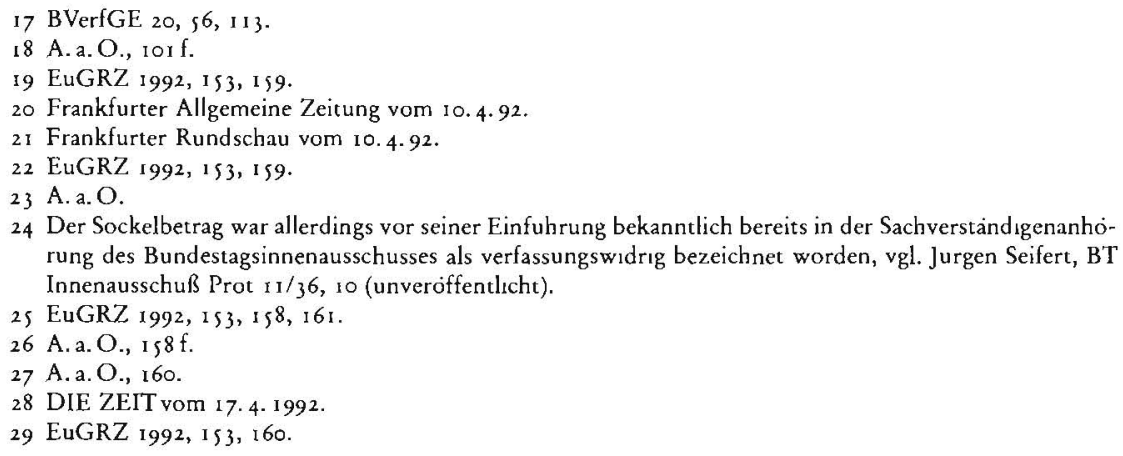


Fortfalls nicht mehr umfassen kann. Allerdings nennt das Gericht als künftigen Richtwert für die bisherige Wahlkampfkostenerstattung die Zahl von 6,50 DM je Wahlberechtigten ${ }^{30}$. Dem jetzigen Modell setzt das Gericht aber einen qualitativ neuartigen Verteilungsmaßstab für die staatlichen Zahlungen an die Parteien entgegen $^{31}$, »in welchen der Erfolg, den eine Partei beim Wähler, den sie bei der Summe der Mitgliedsbeiträge sowie bei dem Umfang der von ihr eingeworbenen Spenden erzielt, zu einem jeweils ins Gewicht fallenden, im einzelnen allerdings vom Gesetzgeber zu bestimmenden Anteil« einfließen soll.

\section{Die Reaktion der Betroffenen}

»Es gibt Urteile aus Karlsruhe, die die Parteien yor ähnliche Schwierigkeiten stellen wie schwere Wahlniederlagen $\ll^{32}$, kommentierte ein Journalist den soeben erörterten Richterspruch. Die Betroffenen verhehlten dagegen unter großen Mühen ihre Überraschung und versuchten in für typisch gehaltener Politikermanier sogleich, die Niederlage in einen Sieg umzudeuten. Bei den offiziellen Einlassungen der Schatzmeister von CDU, CSU, FDP und SPD konnte man fast den Eindruck gewinnen, »als hätten nicht die Grünen, sondern sie selbst die Klage erhoben “ ${ }^{33}$. CDU-Bundesschatzmeister Walther Leisler Kiep hob insbesondere den vom Gericht anerkannten Anspruch der Parteien auf Mitfinanzierung durch den Staat hervor, was auch die Zustimmung der SPD-Schatzmeisterin Inge Wettig-Danielmeier fand, da die Parteien somit nicht länger zu "Wahlfinanzierungsmaschinen« degradiert würden. FDP-Schatzmeister Hermann Otto Solms fand immerhin noch Gefallen daran, daß Spenden in beliebiger Höhe - auch juristischer Personen - weiterhin zulässig sind. Und GRÜNENSchatzmeister Henry Selzer hatte wirklich Grund zur Freude über einen juristischen und politischen Sieg auf ganzer Linie ${ }^{34}$.

Doch selbst DIE GRÜNEN haben sich mit ihrem (auf sie durchschlagenden) Erfolg ins eigene Fleisch geschnitten: Der von ihnen vorrangig mit Hinweis auf die Regionalpartei CSU und die vorgesehene $2 \%$-Klausel, aber nicht prinzipiell in Frage gestellte Sockelbetrag wird als Titel künftig auch in ihrem Parteihaushalt fehlen. Härter werden die Neuregelungen aber die bürgerlichen Parteien treffen, deren Spendenvolumen nach zwischenzeitlichem Rückgang im Jahr der deutschen Einheit (I990) wieder sprunghaft anwuchs. Nach ihren Rechenschaftsberichten nahm die CDU 72,4 Millionen DM, die CSU 35,7 Millionen DM und die FDP ${ }_{2} 3$ Millionen DM an Spenden ein, was Anteilen von $21,9 \%, 39,8 \%$ und $27,5 \%$ an den Gesamteinkünften entspricht ${ }^{35}$. Nun stehe jedoch zu erwarten, »daß die Spendierhosen der Industrie zugenäht werden ${ }^{36}$. Die FDP könnte zudem am ehesten mit der Bestimmung in Konflikt geraten, die eine Finanzierung der politischen Parteien (mindestens) zur Hälfte aus Eigeneinnahmen vorschreibt ${ }^{37}$. Wie die Parteien die neuen Herausforderungen konkret bewältigen wollen, war aus den ersten Stellungnahmen der zuständigen Politiker hingegen nicht zu ersehen: Vorerst wird man weiter im trüben fischen.

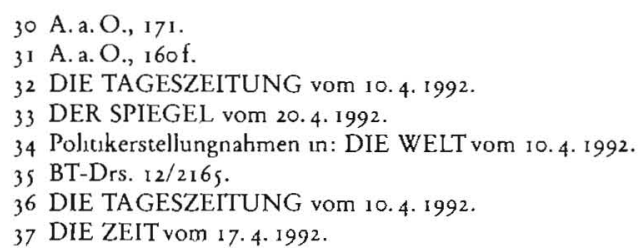


Das Bundesverfassungsgericht hat dem Gesetzgeber aufgegeben, bis Ende 1993 eine Neuregelung zu treffen, so daß die Bundestagswahl r 994 auf verfassungsrechtlich einwandfreier Grundlage im Bereich der Parteienfinanzierung stattfinden könne ${ }^{38}$. Das Gericht empfiehlt an anderer Stelle "die Einschaltung objektiven Sachverstandes «, um ein Korrektiv gegenüber den Insichgeschäften der Politikfinanzierung zu installieren ${ }^{39}$. Ein vergleichbares Gremium sieht bereits $\$ 18$ VIII Parteiengesetz vor, nach dem eine "Kommission unabhängiger Sachverständiger « dem Deutschen Bundestag vor Änderungen in der Struktur und der Höhe der Wahlkampfkostenerstattung Empfehlungen vorlegen soll. Am 23.6. 9992 hat Bundespräsident Richard von Weizsäcker seinen Besetzungsvorschlag für diese Kommission bereits der Öffentlichkeit unterbreitet $4^{\circ}$. Auf solche Expertenrunden setzen auch Michael Vesper und Uwe Günther in einem aktuellen Beitrag zum Thema »Reform der Parteienfinanzierung $\ll^{41}$. Daß diese sich nicht in einem Minimalprogramm erschöpfen kann, welches kaum den Anforderungen des Urteils gerecht wird, machte das Bundesverfassungsgericht selbst deutlich, als es auf den unmittelbaren Zusammenhang zwischen Parteienfinanzierung und Parteienverdrossenheit hinwies ${ }^{42}$. Die CDU sprach sich dementsprechend für ein "glaubwürdigeres, transparenteres und von der Öffentlichkeit akzeptiertes System « aus, indes die FDP die »Hinweise des Gerichts zum Abbau der Entfremdung zwischen Parteien und Bürgern « ernstnehmen will, Bündnis 90/Grüne Politikverdrossenheit abbauen wollen ${ }^{43}$. Diese Aussagen werden jedoch solange Lippenbekenntnisse bleiben, wie die Parteien keine präziseren Vorstellungen artikulieren können.

Das Gericht hat hingegen nicht nur "Kahlschlag im Spendendschungel« betrieben, sondern dem Gesetzgeber auch den Weg in die Zukunft gewiesen. Der Weiterentwicklung der Kleinspendenregelung des $\$ 34 \mathrm{~g}$ Einkommensteuergesetz dürfte kaum etwas entgegenstehen. Das Feld braucht also dort nicht mehr den »Finanzjongleuren und Tricksern « (TAZ) überlassen werden. Bei der an die Stelle der bisherigen Wahlkampfkostenerstattung tretenden Pauschale besteht hingegen einerseits die Gefahr, daß sich die Parteien - unter Hinweis auf den vom Gericht genannten Betrag von $6,50 \mathrm{DM}$ - erneut mit phantasielosem Draufsatteln zufrieden geben werden, daß sie es sich wieder einmal leicht, allzu leicht machen werden. Einige Beobachter befürchten andererseits im Hinblick auf den vom Gericht formulierten, oben bereits angeführten Verteilungsmaßstab die Wiedereinführung von Globalzuschüssen an die Parteien, was einem Rückfall in die soer Jahre gleichkäme, als diese Zuschüsse schon einmal (1959, bis I 966 geübte Praxis) Eingang in den Bundeshaushalt fanden. Damit könnte der angesprochenen Parteienverdrossenheit sicher nicht (erfolgversprechend) begegnet werden. Dafür müßte es vielmehr darauf ankommen, daß »die Entscheidung darüber, ob eine Partei staatliche Leistungen erhält und in welchem Umfang dies geschieht ... beim Bürger selbst verbleibt «44.

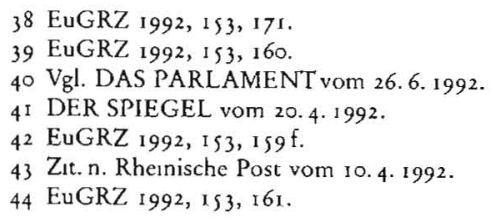


Bereits seit den soer Jahren haben Rechts- und Politikwissenschaft in der Bundesrepublik Deutschland Vorschläge entwickelt, die als prinzipielle "Alternativen zur konventionellen Parteienfinanzierung « zu begreifen sind. Theodor Eschenburg stellte im Bericht der Parteienrechtskommission (1957) erstmals den "Bürgerbeitrag * vor ${ }^{45}$. Hierunter versteht er einen steuerlich abzugsfähigen und für jeden (steuerpflichtigen und wahlberechtigten) Staatsbürger gleichen Betrag, den der Bürger zur Finanzierung der Partei seiner Wahl jährlich »beitragen « kann. Mittels Bürgerbeitrag könnte grundsätzlich auch die gleiche Teilhabe der nicht-steuerpflichtigen Bürger sichergestellt werden. Die dem $\$ 34 \mathrm{~g}$ Einkommensteuergesetz zugrunde liegende Idee müßte dazu nur konsequent zu Ende gedacht werden. In den USA ist im übrigen mit einem ähnlichen Modell ("tax checkoff «) der Beweis erbracht worden, daß der Gesetzgeber den Beitrag so ausgestalten kann, daß die Bürger die erhoffte Resonanz zeigen und die Finanzierung der dortigen Wahlkämpfe gewährleistet ist.

Im Jahre 1983 stellte die vom damaligen Bundespräsidenten Carstens berufene Sachverständigenkommission ihr Gutachten zur Neuordnung der Parteienfinanzierung vor. Mit seinem Bericht präsentierte das Gremium den »Bürgerbonus«, unter dem man einen von der Wahlstimme entkoppelten Finanzbeitrag für die Parteien zu verstehen hat, über dessen Zuweisung die Bürger direkt (mittels "Drittstimme«) zu entscheiden hätten ${ }^{46}$. Der Bonus würde damit die bisherige Art der Wahlkampfkostenerstattung ersetzen können. Mittels dieses plebiszitären Elements der »Finanzwahl « könnte so der Parteienverdrossenheit entgegengewirkt werden, ohne daß Verfassungsbedenken dagegenzustehen haben, da bei der bis dato praktizierten Regelung die Wähler auch schon unmittelbar über die Zuweisung der Wahlkampfkostenerstattung zu befinden haben. Eine detaillierte Untersuchung läßt Bürgerbeitrag und Bürgerbonus insgesamt als verfassungsnähere Alternativen zum gegenwärtigen System der Parteienfinanzierung erscheinen. ${ }^{47}$

45 Bericht der vom Bundesminister des Inneren eingesetzten Parteıenrechtskommission. Rechtliche Ordnung des Parteiwesens, Frankfurt am Main 1958.

46 Bericht zur Neuordnung der Parteienfinanzierung. Vorschlage der vom Bundespräsidenten berufenen Sachverständigenkommission, Koln $1983,217 \mathrm{ff}$;

47 Vgl. hierzu mein Buch »Burgernahe Parteienfinanzierung «, Baden-Baden 1993.

\section{Holger Buck}

\section{Späte Vergeltung oder späte Gerechtigkeit?}

\section{Seit 1989 verfolgt Australien Kriegsverbrecher des "Dritten Reichs"}

\section{Der Anstoß}

"Otherwise we would have been the place where the last Nazis in the world died unpunished and unchased «. Mit diesen Worten kommentiert der Journalist Mark Aarons die jüngst in Australien angelaufene Strafverfolgung von im Land lebenden Kriegsverbrechern der Hitler-Ära. Mark Aarons war es, der im Frühjahr 1987 mit einer fünfteiligen Radio- und einer Fernsehsendung die Aufmerksamkeit der austra- 